Xiao-Ying Huang, Shu-Xiang Sun, Chuan-Qi Wang and Pei Yao*

\title{
Crystal structure of catena-poly[diaqua $\left(\mu_{2}-2-\right.$ (hydroxymethyl)-1 $\mathrm{H}$-imidazole-4,5-dicarboxylato) cadmium(II)], $\mathrm{C}_{6} \mathrm{H}_{8} \mathrm{CdN}_{2} \mathrm{O}_{7}$
}

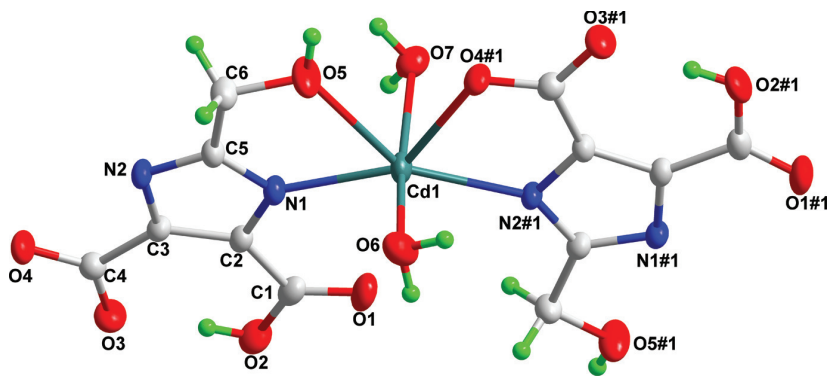

https://doi.org/10.1515/ncrs-2020-0366

Received July 18, 2020; accepted August 21, 2020; available online September 2, 2020

\section{Abstract}

$\mathrm{C}_{6} \mathrm{H}_{8} \mathrm{CdN}_{2} \mathrm{O}_{7}$, monoclinic, $P 2_{1} / c$ (no. 14), $a=7.3428(15) \AA$, $b=11.272(2) \AA, \quad c=12.764(4) \AA, \quad \beta=118.73(2)^{\circ}, \quad V=$ 926.4(4) $\AA^{3}, \quad Z=4, \quad R_{\mathrm{gt}}(F)=0.0261, \quad w R_{\mathrm{ref}}\left(F^{2}\right)=0.0609$, $T=293(2) \mathrm{K}$.

\section{CCDC no.: 2024364}

A part of the molecular structure is shown in the figure $(\# 1=\mathrm{x}, 1.5-\mathrm{y}, 0.5+\mathrm{z})$. Table 1 contains crystallographic data and Table 2 contains the list of the atoms including atomic coordinates and displacement parameters.

\section{Source of material}

All chemical reagents were of analytical purity grade and used without further purification. A mixture of $\mathrm{Cd}\left(\mathrm{NO}_{3}\right)_{2} \cdot 4 \mathrm{H}_{2} \mathrm{O}$ (0.1 mmol), $\mathrm{H}_{4} \mathrm{hmIDC}(0.1 \mathrm{mmol})$, methanol $(2 \mathrm{~mL})$ and distilled water $(2 \mathrm{~mL})$ was sealed in a $25 \mathrm{~mL}$ stainless steel container and heated at $393 \mathrm{~K}$ for $72 \mathrm{~h}$. After the mixture had been

\footnotetext{
*Corresponding author: Pei Yao, School of Chemical and Pharmaceutical Engineering, Changzhou Vocational Institute of Engineering, 213164, Changzhou, Jiangsu, P.R. China, e-mail: yaopeitel@126.com. https://orcid.org/0000-0003-44249492
}

Xiao-Ying Huang, Shu-Xiang Sun and Chuan-Qi Wang: School of Chemical Engineering, Henan Technical Institute, 450042, Zhengzhou, Henan, P.R. China
Table 1: Data collection and handling.

\begin{tabular}{ll}
\hline Crystal: & Yellow prism \\
Size: & $0.21 \times 0.20 \times 0.18 \mathrm{~mm}$ \\
Wavelength: & Mo $K \alpha$ radiation $(0.71073 \AA)$ \\
$\mu:$ & $2.38 \mathrm{~mm}^{-1}$ \\
Diffractometer, scan mode: & Rigaku Saturn, $\omega$ \\
$\theta_{\max }$, completeness: & $28.0^{\circ},>99 \%$ \\
$N(h k l)_{\text {measured }}, N(h k l)_{\text {unique }}, R_{\text {int }}:$ & $11145,2218,0.023$ \\
Criterion for $I_{\text {obs }}, N(h k l)_{\text {gt }}:$ & $I_{\text {obs }}>2 \sigma\left(I_{\text {obs }}\right), 2179$ \\
$N(\text { param })_{\text {refined }}:$ & 146 \\
Programs: & Rigaku $[1]$, SHELX [2, 3] \\
\hline
\end{tabular}

Table 2: Fractional atomic coordinates and isotropic or equivalent isotropic displacement parameters $\left(\AA^{2}\right)$.

\begin{tabular}{lrrrr}
\hline Atom & $\boldsymbol{x}$ & $\boldsymbol{y}$ & $\boldsymbol{z}$ & $\boldsymbol{U}_{\text {iso }}{ }^{*} \boldsymbol{U}_{\text {eq }}$ \\
\hline Cd1 & $0.76875(3)$ & $0.68699(2)$ & $0.60990(2)$ & $0.02556(8)$ \\
N1 & $0.7686(4)$ & $0.7849(2)$ & $0.46006(19)$ & $0.0229(4)$ \\
N2 & $0.7648(4)$ & $0.80884(18)$ & $0.28531(19)$ & $0.0219(4)$ \\
O1 & $0.7577(3)$ & $0.9575(2)$ & $0.61427(17)$ & $0.0374(5)$ \\
O2 & $0.7387(4)$ & $1.10193(18)$ & $0.49297(19)$ & $0.0351(5)$ \\
H2 & 0.7307 & 1.1083 & 0.4269 & $0.042^{*}$ \\
O3 & $0.7292(4)$ & $1.12567(17)$ & $0.29964(19)$ & $0.0335(5)$ \\
O4 & $0.7522(3)$ & $1.01189(17)$ & $0.16483(17)$ & $0.0294(4)$ \\
O5 & $0.7598(4)$ & $0.54989(19)$ & $0.4501(2)$ & $0.0448(6)$ \\
H5A & 0.7379 & 0.4788 & 0.4359 & $0.054^{*}$ \\
O6 & $1.1297(3)$ & $0.6522(2)$ & $0.7099(2)$ & $0.0383(5)$ \\
H1W & 1.1674 & 0.7167 & 0.7491 & $0.046^{*}$ \\
H2W & 1.1516 & 0.6036 & 0.7657 & $0.046^{*}$ \\
O7 & $0.4021(3)$ & $0.67143(17)$ & $0.51172(19)$ & $0.0321(5)$ \\
H3W & 0.3730 & 0.7423 & 0.4861 & $0.039^{*}$ \\
H4W & 0.3657 & 0.6347 & 0.4465 & $0.039^{*}$ \\
C1 & $0.7494(4)$ & $0.9896(3)$ & $0.5212(2)$ & $0.0263(5)$ \\
C2 & $0.7543(4)$ & $0.9035(2)$ & $0.4361(2)$ & $0.0218(5)$ \\
C3 & $0.7518(4)$ & $0.9184(2)$ & $0.3279(2)$ & $0.0210(5)$ \\
C4 & $0.7445(4)$ & $1.0247(2)$ & $0.2591(2)$ & $0.0239(5)$ \\
C5 & $0.7736(4)$ & $0.7328(2)$ & $0.3676(2)$ & $0.0225(5)$ \\
C6 & $0.7877(5)$ & $0.6018(2)$ & $0.3587(3)$ & $0.0281(6)$ \\
H6A & 0.6813 & 0.5742 & 0.2813 & $0.034^{*}$ \\
H6B & 0.9224 & 0.5802 & 0.3677 & $0.034^{*}$ \\
\hline & & & &
\end{tabular}

allowed to cool to room temperature at a rate of $5 \mathrm{~K} \mathrm{~h}^{-1}$, light yellow crystals of $\mathrm{Cd}\left(\mathrm{H}_{2} \mathrm{hmIDC}\right)\left(\mathrm{H}_{2} \mathrm{O}\right)_{2}$ were obtained (yield $49 \%$, based on $\mathrm{Cd}$ ). 


\section{Experimental details}

Hydrogen atoms on carbon atoms were positioned geometrically and refined as riding atoms, with $\mathrm{C}-\mathrm{H}=0.97 \AA$ A. Hydrogen atoms of the nondeprotonated carboxylic acid groups of $\mathrm{H}_{2} \mathrm{hmIDC}^{2-}$ and hydroxyl groups $(-\mathrm{OH})$ were refined as riding atoms, with $\mathrm{O}-\mathrm{H}=0.82 \AA$. Hydrogen atoms of the water molecules were located in a difference Fourier map and the $\mathrm{O}-\mathrm{H}$ distance constrained to $0.85 \AA$ A.

\section{Comment}

$N$-heterocyclic carboxylic acids have been widely used as ligands since they can offer different donors and diverse coordination modes due to the existence of carboxylate groups as well as potential $N$-donors. So far, complexes with various topologies and potential applications have been synthesized [4-9]. $\mathrm{N}$-heterocyclic carboxylic acid, 2-(hydroxymethyl)- $1 H$-imidazole-4,5-dicarboxylic acid $\left(\mathrm{H}_{4} \mathrm{hmIDC}\right)$ is a good linker since it has two potential $\mathrm{N}$-donors and five potential $\mathrm{O}$-donors and can coordinate to almost all soft and hard metal ions with various coordination modes. Up to now, several complexes based on $\mathrm{H}_{\mathrm{x}} \mathrm{hmIDC}$ ligand have been reported [6, 7, 10-12].

The asymmetric unit of the title structure consists of one $\mathrm{Cd}(\mathrm{II})$ cation, one dianionic $\mathrm{H}_{2}$ hmIDC $^{2-}$ ligand and two coordinating water molecules. Each $\mathrm{Cd} 1$ ion is six-coordinated and located in a distorted octahedral $\mathrm{CdN}_{2} \mathrm{O}_{4}$ coordination environment formed by two $\mathrm{N}$ atoms ( $\mathrm{N} 1$ and $\mathrm{N} 2 \# 1)$ and two $\mathrm{O}$ atoms (O4\#1 and 05) from two $\mathrm{H}_{2} \mathrm{hmIDC}^{2-}$ anions and by two water molecules ( $\mathrm{O} 6$ and $\mathrm{O7}$ ). The $\mathrm{Cd}-\mathrm{O}$ bond lengths range from 2.357(2) to 2.535(2) $\AA$ and the $\mathrm{Cd}-\mathrm{N}$ bond lengths are 2.207(2) and 2.254(2) $\AA$, respectively; these values are within the normal ranges and close to those reported in other $\mathrm{Cd}(\mathrm{II})$ complexes [13-15]. Cd(II) ions are linked by $\mathrm{H}_{2} \mathrm{hmIDC}^{2-}$ ligand into one-dimensional chains that run along the $c$ axis. The intra-chain $\mathrm{Cd}(\mathrm{II})-\mathrm{Cd}(\mathrm{II})$ distance is 6.538(2) Å. In addition, there are $\mathrm{O}-\mathrm{H} \cdots \mathrm{O}$ intramolecular hydrogen bonds between carboxyl and carboxylate groups, and five $\mathrm{O}-\mathrm{H} \cdots \mathrm{O}$ intermolecular hydrogen bonds involving hydroxyl groups, carboxyl groups, carboxylate groups and water molecules. Adjacent chains are linked by the hydrogen bonds mentioned above, leading to a three-dimensional network.

Acknowledgements: This study was supported by Qinglan Project of Jiangsu Province (2020), Science and Technology Innovation Team of Changzhou Vocational Institute of
Engineering (2020); Postgraduate Research \& Practice Innovation Program of Jiangsu Province (KYCX17_1876); Changzhou science and technology support plan (CE20195007).

\section{References}

1. Rigaku/MSC. CrystalClear. Rigaku/MSC Inc., The Woodlands, Texas, USA (2006).

2. Sheldrick, G. M.: A short history of SHELX. Acta Crystallogr. A64 (2008) 112-122.

3. Sheldrick, G. M.: Crystal structure refinement with SHELXL. Acta Crystallogr. C71 (2015) 3-8.

4. Azouzi, K.; Hamdi, B.; Zouari, R.; Salah, A. B.: Synthesis, structure and Hirshfeld surface analysis, vibrational and DFT investigation of (4-pyridine carboxylic acid) tetrachlorocuprate (II) monohydrate. Bull. Mater. Sci. 40 (2017) 289-299.

5. Liu, Y. J.; Cheng, D.; Li, Y. X.; Meng, X. R.; Yang, H. X.: A new two-dimensional managnese(II) coordination polymer constructed by 2,2'-(1,2-phenylene)bis( $1 \mathrm{H}$-imidazole-4, 5-dicarboxylate). Acta Crystallogr. C74 (2018) 599-603.

6. Cheng, D.; Liu, Y. J.; Cheng, F. R.; Yang, H. X.; Meng, X. R.: Synthesis, structure and fluorescence properties of a $\mathrm{Zn}$ (II) coordination polymer based on 2-(hydroxymethyl)- $1 \mathrm{H}$-imidazole-4,5dicarboxylate. J. Chem. Res. 490 (2018) 490-493.

7. Yang, H. X.; Jian, S. J.; Liang, Z.; Zhang, J. D.; Meng, X. R.: The first 3D Zn(II) complex involving chiral chains constructed by achiral ligand 2-(hydroxymethyl)-1H-imidazole4,5-dicarboxylate. Inorg. Chem. Commun. 61 (2015) 57-59.

8. Shao, Z. C.; Meng, X. R.; Hou, H. W.: Effect of pH on the construction of $\mathrm{Cd}^{\prime \prime}$ coordination polymers involving the $1,1^{\prime}$ - $[1$, 4-phenylenebis(methylene)]bis-(3,5-dicarboxylatopyridinium) ligand. Acta Crystallogr. C75 (2019) 1142-1149.

9. Li, T.; Xiu, Y.; Su, X.; Meng, X. R.: Syntheses, crystal structures, and fluorescent properties of two Cd(II) complexes based on 2,2'-(ethane-1,2-diyl)bis(1H-imidazole-4,5-dicarboxylic acid). J. Coord. Chem. 65 (2012) 3111-3121.

10. Cai, S.-L.; Zheng, S.-R.; Wen, Z.-Z.; Fan, J.; Zhang, W.-G.: Construction of $\mathrm{Ba}$ (II) coordination polymers based on imidazolebased dicarboxylate ligands: structural diversity tuned by alcohol solvents. Cryst. Growth Des. 12 (2012) 3575-3582.

11. Zheng, S.-R.; Cai, S.-L.; Pan, M.; Fan, J.; Xiao, T.-T.; Zhang, W.-G.: The construction of coordination networks based on imidazole-based dicarboxylate ligand containing hydroxymethyl group. CrystEngComm 13 (2011) 883-888.

12. Li, T.-T.; Cai, S.-L.; Zeng, R.-H.; Zheng, S.-R.: Structures and luminescent properties of two new main group coordination polymers based on 2-(hydroxymethyl)- $1 \mathrm{H}$-imidazole-4, 5-dicarboxylic acid. Inorg. Chem. Commun. 48 (2014) 40-43.

13. Huang, Q.-Y.; Lin, X.-Y.; Meng, X.-R.: A new one-dimensional Cd" coordination polymer with a two-dimensional layered structure incorporating 2-[(1H-imidazol-1-yl)methyl]- $1 H$ benzimidazole and benzene-1,2-dicarboxylate ligands. Acta Crystallogr. C72 (2016) 480-484. 
14. Yang, Y. Q.; Su, C. F.; Zhang, J. D.; Yang, H. X.; Zhang, G. Y.; Meng, X. R.: Construction of Cd(II) complexes based on 2-(1H-imidazol-1-methyl)-1H-benzimidazole and 1,4-benzenedicarboxylate. J. Coord. Chem. 69 (2016) 3762-3775.
15. Li, X.-F.; Ma, L.-G.; Yang, Y.-Q.; Liu, Y.-J.; Meng, X.-R.; Yang, H.-X.: Synthesis, crystal structure and bovine serum albumin-binding studies of a new Cd(II) complex incorporating 2,2'-(propane-1,3-diyl) bis(1H-imidazole-4,5-dicarboxylate). J. Chem. Res. 44 (2020) 198-205. 